\title{
McLeod neuroacanthocytosis syndrome
}

INSERM

\section{Source}

INSERM. (1999). Orphanet: an online rare disease and orphan drug data base. McLeod neuroacanthocytosis syndrome. ORPHA:59306

McLeod neuroacanthocytosis syndrome (MLS) is a form of neuroacanthocytosis (see this term) and is characterized clinically by a Huntington's disease-like phenotype with an involuntary hyperkinetic movement disorder, psychiatric manifestations and cognitive alterations, and biochemically by absence of the Kx antigen and by weak expression of the Kell antigens. 